\title{
La intervención motivacional y sus efectos sobre la entrada a tratamiento de adicción
}

\section{Motivational intervention and its effects on addiction treatment entry}

\author{
Cristián Lópeza ${ }^{a}$ Caterina Bruzzone ${ }^{b}$, Mariana Krebs ${ }^{c}$ y Ximena Castro ${ }^{d}$ \\ a Pontificia Universidad Católica de Chile, Santiago, Chile ${ }^{\mathrm{b}}$ Grupo Crisol, Santiago, Chile \\ ${ }^{\mathrm{c}}$ Centro de Salud Familiar UC Madre Teresa de Calcuta, Santiago, Chile ${ }^{\mathrm{d}}$ Conocimiento Activo Consultores, Santiago, Chile
}

\begin{abstract}
Resumen
Se presentan los resultados de un estudio de caso acerca de las vivencias de sujetos que participaron en un proceso de intervención motivacional (IM) de seis semanas de duración, que incluye la participación de terceros significativos. Se discute la importancia de incluir la llamada muestra de abstinencia en una intervención que busque motivar a la entrada a un tratamiento de adicción. Los sujetos participantes son seis adultos, con edades entre 25 y 45 años, de sexo masculino, que habían participado de un modo de intervención específico llamado intervención motivacional, que incluye la muestra de abstinencia. A partir de un análisis cualitativo de los datos, se describen los cambios producidos durante este proceso y la forma en que estos cambios facilitan que los sujetos tomen la decisión de tratarse. Los resultados muestran que la muestra de abstinencia, realizada en el contexto de una intervención con las características de la IM, tienen un efecto positivo en la decisión de los sujetos de entrar a un tratamiento. Finalmente, se remarca la importancia de ofrecer un modo de intervención que lleve a que el sujeto se sienta parte activa del proceso de cambio.
\end{abstract}

Palabras clave: adicción, abstinencia, motivación, terceros significativos, tratamiento.

\begin{abstract}
The results of a case study regarding the experiences of individuals who participated in a motivational intervention (MI) process for a six week period which includes the participation of significant others are presented. The importance of including the so called sobriety sampling in an intervention that looks for motivating the entrance to an addiction treatment is discussed. The individuals who participated are six male adults aged between 25 and 45 , who had already participated on a specific mode of intervention called motivational intervention (MI), which includes the sobriety sampling. The changes produced during this process are described based on qualitative analysis of the data. Results show that the sobriety sampling, in the context of an intervention with the characteristics of the MI, makes it easier for individuals to take the decision to treat themselves. Finally, the importance of offering a mode of intervention in which the subject is an active part of the process of change is highlighted.
\end{abstract}

Keywords: addiction, abstinence, motivation, significant others, treatment.

Contacto: C. López, Avda.Vicuña Mackenna 4860, Macul, Santiago. cristian@uc.cl

Cómo citar este artículo:

López, C., Bruzzone, C., Krebs, M., y Castro, X. (2013). La intervención motivacional y sus efectos sobre la entrada a tratamiento de adicción. Revista de Psicología, 22(1), 37-47. doi: 10.5354/0719-0581.2013.27717 


\section{Introducción}

La posibilidad de tratar a pacientes que han desarrollado una adicción a sustancias se encuentra con dos obstáculos iniciales. Por una parte, es solo un porcentaje minoritario de la población el que consulta alguna vez en su vida (Cunningham y Breslin, 2004; Stinson, Grant, Dawson, Ruan, Huang y Saha, 2005; Substance Abuse and Mental Health Services Administration, 2009); y por otra parte, de este porcentaje una gran mayoría lo hace empujado por terceros significativos (Wild, Cunningham y Ryan, 2006). Esto ha llevado a que la noción de motivación se convierta en un elemento importante al momento de conceptualizar las intervenciones en estos casos (Beck, Wright, Newmann y Liese, 1993/1999; Carpenter, Miele y Hasin, 2002; Di Clemente, Bellino y Neavins, 1999; Downey, Rosengren y Donovan, 2001; Lincourt, Kuettel, y Bombardier, 2002; Miller y Rollnick, 1991/1999; Ulivi, 2000). Desde esta noción de motivación se han propuesto estrategias de intervención muy distintas, tanto en los supuestos asumidos con respecto al funcionamiento de la adicción, la lógica de intervención seguida, como en los objetivos planteados, y en las instancias terapéuticas incluidas (Johnson, 1973; Kaskutas, Subbaraman, Witbrodt y Zemore, 2009; Landau et al., 2004; Lee et al, 2012; Meyers, Roozen, y Smith, 2011; Miller y Rollnick, 1991/1999; Santisteban, Suárez-Morales, Robbins y Szapocznik, 2006; Tatarsky, 2003; Timko y DeBenedetti, 2007).

En varias de las estrategias de intervención motivacionales utilizadas destaca la incorporación de terceros significativos como un modo de llevar al sujeto a que decida cambiar su relación con las sustancias adictivas. Otro elemento, más específico, que también ha sido utilizado es la llamada muestra de abstinencia (Meyers y Smith, 1995; Miller, Forcehimes y Zweben, 2011; Miller y Meyers, 1999; Miller y Page, 1991). La muestra de abstinencia consiste en pactar un tiempo de abstinencia que permita comenzar a realizar un cambio sin un compromiso mayor de tratarse por parte del sujeto. Se ha planteado esta posibilidad como una alternativa para el ingreso a tratamiento, ya que produciría menos resistencia en el sujeto que la petición de una abstinencia en el largo plazo.

Existe una forma específica de abordaje motivacional ambulatorio llamada intervención motivacional (IM), que, junto a otro conjunto de elementos utiliza la muestra de abstinencia como elemento central de su lógica de funcionamiento. El modelo de la IM se desarrolló a partir del abordaje llamado Entrevistas Previas (Kreither y López, 2001), y ha sido utilizado en distintos contextos terapéuticos en Chile. La IM consiste en una serie de entrevistas psicológicas y, eventualmente psiquiátricas, durante un período de seis semanas. En estas entrevistas participan tanto el sujeto con el supuesto problema de consumo de sustancias, como los terceros significativos que lo acompañan, existiendo instancias terapéuticas individuales e instancias familiares. Eventualmente se puede incluir el uso de disulfiram tomado en forma asistida, y la realización de exámenes de orina para detectar el uso de sustancias adictivas. El proceso comienza con una primera entrevista, a la cual normalmente se solicita que vayan todos los involucrados en la consulta, en la que se intenta llegar al acuerdo de realizar estas seis semanas de IM. El acuerdo de realizar estas seis semanas incluye el que el sujeto intente seriamente, durante ese período, estar en abstinencia del consumo de cualquier sustancia adictiva, a modo de prueba. La IM concluye con una entrevista con todos los que participaron del proceso, en la cual se discute la posibilidad de que el sujeto ingrese a un tratamiento de adicción propiamente tal, llegándose a una decisión al respecto.

La IM tiene, entonces, dos elementos centrales: (1) la incorporación de terceros significativos a la discusión de las alternativas de decisión y acción que tiene el sujeto; (2) la realización de un acuerdo con el sujeto, y los terceros significativos participantes, de realizar un período de muestra de abstinencia, consistente en que el sujeto intente estar sin consumo de sustancias adictivas durante seis semanas que dura el proceso, a la vez que se comienza a tener reuniones individuales y familiares. El primero de los elementos tiene un fuerte fundamento empírico, ya que como se planteaba anteriormente, la gran mayoría de los pacientes consulta movilizado por terceros significativos, marcando la incorporación de estos una importante diferencia en el resultado. El segundo de los elementos se basa en el hecho que el consumo de sustancias adictivas produce una serie de efectos sobre la situación en la que se encuentra el sujeto, que dificultan la toma y mantención de una decisión de tratarse. En este punto son interesantes los planteamientos de Heyman (2009), quien cuestiona las definiciones tradicionales de la adicción e intenta precisar qué es lo que caracteriza a las sustancias adictivas. Concluye que estas son especiosas, que no llevan a la retroalimentación auto-inhibitoria connatural al resto de las actividades, que son intoxicantes y que impiden un análisis costo-beneficio basado en una elección global. Las dos primeras serían particulares a las sustancias adictivas. La característica de especiosa alude a que involucran una recompensa de alto valor debido a la especial cualidad e intensidad de la satisfacción, a que esta es inmediata y a que los costos son desplazados hacia el futuro. El que no llevan a una retroalimentación auto-inhibitoria, significa que las sustancias adictivas no producen lo que normalmente sí ocurre, es decir, que la recurrencia de una actividad conduzca al sujeto al aburrimiento, a la fatiga o la saciedad, disminuyendo así el valor de recompensa. De esta manera, el valor de recompensa relativo al resto de las alternativas de gratificación 
disponibles es cada vez mayor, quedando el sujeto cada vez más atrapado en una única forma de gratificación. Si a lo anterior se agrega el que las sustancias son intoxicantes e impiden un análisis costo-beneficio global, se entiende que una detención de su consumo por un período puede ser clave para dar la oportunidad de que se generen nuevas condiciones en la situación del sujeto que le permitan tomar una decisión de tratarse.

La IM, entonces, opera desde una lógica similar a la de las aproximaciones estratégicas en psicoterapia, en el sentido que busca interrumpir el ciclo repetitivo de perpetuación del problema a través de la instauración de un mínimo cambio con el cual se pueda lograr una reacción en cadena que termine modificando todo el sistema (Nardone y Watzlawick, 1990/1992). Además, esta meta se instaura en base a un acuerdo con todos los involucrados en la consulta, planteándosela de un modo concreto y claro, e incluyendo elementos como frecuencia de las intervenciones, duración del proceso y lugar en el cual se llevará a cabo (Cade y O'Hanlon, 1995). Desde una aproximación estratégica se esperaría que una intervención efectiva genere en el paciente una reducción sintomática ya en los inicios del proceso, y produzca un cambio progresivo de la percepción de sí mismo, de los otros y del mundo. Dicho proceso se manifestaría en un desplazamiento gradual de la rigidez (típica del sistema que mantenía el problema), hacia una percepción más flexible de la realidad, con un aumento progresivo de la autonomía personal y autoestima dada la constatación de la posibilidad de resolver el problema (Watzlawick y Nardone, 2000).

Con la IM se espera, entonces, cortar una retroalimentación positiva entre la conducta de consumo y el no lograr decidir nada con respecto a la posibilidad de hacer algo con el problema, a través de un período de muestra de abstinencia de seis semanas que incorpora a los terceros significativos. El corte de esta retroalimentación se relaciona concretamente con la desintoxicación del sujeto, pero también con el mensaje que se le transmite, a él y a los terceros significativos, de que es capaz de detener el consumo si así lo decide. El hecho que la IM se haga de manera ambulatoria lleva a que la detención del consumo sea el efecto de un trabajo en conjunto entre el sujeto que decide realizar esta prueba, y los terceros significativos que lo ayudan con algunos apoyos concretos durante las seis semanas. Esto produce una situación en que inevitablemente se tendrán que desarrollar confianzas y auto-confianzas en los integrantes del sistema que consulta, cambiándose pautas de relación y de auto-percepción propias de este tipo de problema. Los objetivos a alcanzar con la IM son: que los cambios producidos en el sujeto y su sistema interaccional lleven a que el sujeto decida aceptar entrar en un tratamiento de adicción; y lograr una cierta apertura del sujeto que le permita quedar mejor predispuesto para la realización de un trabajo psicoterapéutico desde la aproximación terapéutica que se defina. De esta manera, por una parte se sigue una lógica estratégica y, por otra, se intenta sentar las bases para la entrada en un proceso terapéutico de cualquier línea teórica, por lo que también se ha podido entender lo realizado en la IM desde la lógica de las entrevistas preliminares de Lacan (López, 2006).

En este artículo se plantearán los resultados de un estudio que busca investigar sobre el efecto que tiene la realización de una muestra de abstinencia en los sujetos que pasan por este proceso de seis semanas de IM, la cual implica un contexto con una importante participación de los terceros significativos. También interesará entender cómo estos efectos se enlazan con la decisión del sujeto de entrar a un tratamiento de adicción de mediano plazo, que pueda incluir un trabajo psicoterapéutico. La investigación se lleva a cabo a partir del análisis del relato de las vivencias de los sujetos participantes. Los resultados mostrados en este artículo son complementarios a otros, de un estudio diferente, referidos a la IM (López, 2009, 2011a).

\section{Metodología}

\section{Participantes}

Los sujetos fueron escogidos de un grupo de pacientes con diagnóstico de adicción a sustancias químicas que habían ingresado a un programa de tratamiento, luego de haber participado de un proceso de intervención motivacional de seis semanas de duración. El diagnóstico de adicción había sido realizado por los profesionales (psicólogos y psiquiatra) a cargo del programa al momento de llegar a consultar los pacientes, de acuerdo a los criterios del DSM-IV. Los sujetos escogidos no tenían diagnóstico dual. El programa de tratamiento se realizaba en Chile, $y$ todos los participantes eran chilenos.

La muestra para esta investigación ha sido seleccionada en base a la estrategia de muestreo de casos críticos o relevantes propuesta por Patton, que consiste en seleccionar casos que permitan una mejor comprensión de la realidad en estudio, dado que su relación con esta última es especialmente clara (Patton, 1990, citado en Flick, 2002/2004). Los criterios de relevancia considerados fueron: (1) haber pasado por el período completo de IM ambulatorio; (2) haber cumplido por lo menos un año de abstinencia completa (pudiendo incluir hasta dos recaídas puntuales, es decir, de no más de un día de duración) y haber egresado hace no más de tres años del programa, encontrándose actualmente en abstinencia 
completa. El criterio de un año de abstinencia se utilizó con el fin de que los sujetos no estuvieran interferidos por el vínculo adictivo con la sustancia en su percepción de lo vivido. No fue posible seleccionar a pacientes que se encontraran exactamente en el mismo momento de su tratamiento. La amplitud del rango de tiempo se definió con la finalidad de homogeneizar, en la medida de lo posible, el momento en el que se encuentran los pacientes, para que este factor no explique las diferencias entre los relatos.

Un criterio de relevancia que hubiese sido interesante incorporar es el de haber realizado la IM y haber decidido no entrar a tratamiento. Sin embargo, no se cuenta con pacientes que cumplan con dicho criterio, dado que quienes realizaron el periodo de IM decidieron iniciar tratamiento.

Considerando los criterios de relevancia mencionados, el tamaño de la muestra fue de seis sujetos. De estos, cuatro se encontraban en su segundo año de tratamiento y los otros dos habían egresado hacía dos años y medio. Los sujetos escogidos estaban, al momento de pasar por la IM, en un rango de edad entre 25 y 45 años, con un promedio de 34.5 años. Son de un nivel socioeconómico medio-alto, tres de ellos tenían estudios superiores técnicos, dos tenían enseñanza media completa y uno era profesional universitario. Al momento de llegar a consultar, cuatro de los sujetos tenían un trabajo estable, dos eran solteros, dos casados y dos convivían con sus parejas. En relación a las sustancias consumidas, cuatro de ellos consultaron por consumo de alcohol, marihuana y cocaína, y dos solo por alcohol. Dos de los sujetos tuvieron al menos una recaída durante el período de seis semanas, y cuatro de ellos no tuvieron recaídas.

\section{Procedimiento de recolección de datos}

Con cada uno de los sujetos se llevó a cabo una entrevista episódica (Flick, 2000), que tuvo una duración de entre una, y una hora y media. La entrevista fue realizada por dos psicólogas, estudiantes de magister. Una de ellas no conocía a los pacientes, y la otra había participado en algunas instancias del programa de tratamiento. En esta entrevista se les pidió a los sujetos que hablaran libremente del recuerdo que tenían de su experiencia en relación a las seis semanas de la IM, y también se le hizo preguntas para intencionar su reflexión hacia determinados elementos del proceso que eran de interés para el estudio.

Se escogió este instrumento ya que se pretende recoger tanto el conocimiento episódico del sujeto, que se organiza más cerca de las experiencias vividas por este, como el conocimiento semántico, basado en relaciones abstraídas a partir de sus vivencias. Para acceder a ambas formas de conocimiento el instrumento emplea las narraciones y las preguntas intencionadas concretas, respectivamente. Por tanto, este instrumento posibilita explotar las ventajas tanto de la entrevista narrativa como de la semi-estructurada, ya que permite acercarse al mundo experiencial del entrevistado, al mismo tiempo que el entrevistador puede entablar un diálogo natural, bajo el esquema pregunta-respuesta, que permite acceder a especificidades de la construcción del entrevistado. Es así como se logra conocer tanto la vivencia construida por el entrevistado, como los aspectos comunes y las diferencias entre las visiones y las construcciones semánticas de los entrevistados. Lo anterior constituye una triangulación de los enfoques de recogida de datos, pertinente para el presente estudio (Flick, 2002, 2004).

La entrevista se realizó en las dependencias del centro donde funciona el programa, y contó con el consentimiento informado de los participantes para utilizar la información con fines de investigación. La confidencialidad del material se aseguró borrando cualquier información que pudiera hacer reconocible al individuo particular. No hubo personas que se rehusaran a participar.

Luego de realizadas las seis entrevistas y de un primer análisis del material obtenido, se evaluó si era necesario agregar otras entrevistas, ya sea a otros sujetos o a los mismos, pero no se consideró necesario, ya que aparecía una saturación del material. Los sujetos entrevistados no revisaron las transcripciones ni el análisis realizado.

\section{Análisis de datos}

El análisis de los datos recogidos se realizó mediante la estrategia de codificación, con el propósito de categorizar el material en función de las vivencias que fue posible distinguir a partir del relato de los entrevistados. Más específicamente se empleó la codificación teórica y la codificación temática, aplicables al material que aporta la entrevista episódica, y acordes a los propósitos del presente estudio, ya que permiten rescatar cada caso individual y, en un segundo paso, desarrollar un esquema analítico común (Flick, 2002, 2004). En esta codificación participaron las dos psicólogas que realizaron las entrevistas, sin apoyo de software.

En cuanto a la codificación temática, esta permite comparar las visiones recogidas a partir de un análisis profundo de los relatos individuales, generando categorías y derivando la estructura de relaciones propia de cada caso individual. Así se favorece la comparación posterior y la comprensión de la manera en que cada sujeto se enfrenta al problema de estudio. La codificación teórica, por su parte, permite segmentar los datos recogidos en códigos más abstractos, que posibilitan comprender los relatos, analizando sus similitudes y 
diferencias, en función de la pregunta de investigación, para llegar a un planteamiento de los elementos centrales comunes a los sujetos entrevistados (Flick, 2002, 2004).

\section{Resultados}

Los resultados serán ilustrados a través de extractos de las entrevistas realizadas para efectos de este estudio.

Un primer elemento que aparece claramente asociado a la realización de la muestra de abstinencia es la emergencia de una sensación de bienestar en los sujetos. Este bienestar se manifiesta de diversos modos. Así, se puede distinguir un bienestar emocional relacionado con el término de una doble vida que se había estado llevando:

- Emocionalmente estai espectacular, te sentís re bien, es otra vida... yo creo que al terminar la doble vida te sentís re bien, emocionalmente, ahí es como ah! qué alivio, contar todo, no estar ocultando nada, eso te ayuda n, te fortalece harto.

También aparece un bienestar físico, muy valorado por los sujetos:

- Volviendo a esas seis semanas, yo creo que lo que... mi bienestar físico fue fundamental, o sea, el hecho de dejar de consumir te hace sentir bien físicamente, estás más despierto y eso te hace darte cuenta de que puedes, y así la segunda semana, y a la otra vai a estar mejor, y a la otra mejor.

- Yo quería seguir dándole... en las seis semanas me fui sintiendo cada día mejor, en las relaciones, yo como persona, cada día que pasaba me levantaba con más ganas.

- Con esa abstinencia, el cambio físico que se percibe es notorio, es súper notorio, no sé, te cambia el color de la piel, te cambia el olor del cuerpo, te cambia la manera de hablar, la manera de actuar, en todo sentido, o sea, en seis semanas nada más.

Junto al bienestar físico también se manifiesta una mayor claridad cognitiva:

- Yo diría que fue una especie de... todo ese tiempo fue como despertar de un sueño, como volver a recordar cosas, volver a adquirir habilidades que había perdido, como tener más claridad en el pensamiento, era como te digo, como despertar de un sueño... y adquirir un poco.. de 1 a 100, un $1 \%$ más de claridad en la cabeza.

Se produce una disminución de los deseos de consumo que también contribuye a la sensación de bienestar: "Este síndrome de abstinencia, después ya va pasando, ya después no me sentía tan mal, porque yo creo que a la semana yo ya no necesitaba tanto consumir".
A las emergentes sensaciones de bienestar se les suma que el lograr mantener la abstinencia va produciendo una mayor percepción de autoeficacia:

- De repente yo empecé a ser otro Benjamín en el trabajo, me lo dijeron montones de personas, porque yo no era así antes ... porque a las seis semanas yo me di cuenta de que no, de que podía estar sin consumir, que eso era lo más difícil, que podía estar sin consumir... como ya puedo.

- Pero lo más importante es ir viendo que puedes, que puedo estar en abstinencia, eso es súper importante, yo creo que ya la segunda semana como que uno toma conciencia de que ya puedes estar 5 días, o 6 días, o 7 días sin consumir, entonces puedo estar 7 días más, y ya no son tan difíciles como los primeros 7 días, cada vez van siendo menos difíciles.

- Lo que me lleva a seguir es que se puede salir... que surge la idea de que se puede hacer algo, que quizás no está todo perdido, que quizás la huevá no es tan loca.

- Yo creo que me reafirmó caleta, en el sentido de poder mantenerme abstinente, por las mías, yo creo que eso me dio el... impulso necesario como pa' decir, puedo, o podría llegar a hacerlo... no sé... el igual lo voy a hacer.

El mayor bienestar y la mayor percepción de autoeficacia llevan a tener una perspectiva más positiva frente a las situaciones y dificultades vitales:

- Porque gran parte de mis problemas se me arreglaron cuando dejé de consumir.

- El estar lúcido me ayudó a... o yo los veía más grande en este tiempo de lo que eran [los problemas], eh.. igual yo soy como bien dramático con los problemas, como melodramático, entonces, claro, después me ayudó a verlos un poco más tranquilo.

- Y seguir con la idea de que sí lo voy a lograr, de que sí se podía hacer algo, de que de repente la vida sí tenía sentido, de que de repente huevón... puta... había un camino que de repente valía la pena tomarlo... y de repente no estaba todo tan perdido.

Los sujetos relatan una mejoría significativa en las relaciones con su familia durante el período de muestra de abstinencia:

- En las seis semanas me fui sintiendo cada día mejor, en las relaciones... como te digo, en ese mes yo vi un cambio en mi familia.

- Entonces en las seis semanas, vas viendo frutos... vas viendo que puedes salir... ya tienes otro apoyo, vas viendo más confianza en tu casa.

- El haber estado en la casa, con los niños, jugar con los cabros chicos, entonces ahí me di cuenta que tenía que seguir, porque antes no había estado.

- O sea, se me empezaron a dar las cosas familiares, o sea, hubo reuniones enteras de familia, tanto de mamá, como 
hijos, mis hermanos empezaron a funcionar mucho mejor, mi mamá también, o sea yo vi un cambio radical en mi familia, o sea del estado de depresión en la que estaban como que empezaron a vivir la vida de nuevo, con el Gabriel cambiado, entonces yo dije no, como que tengo que hacerlo, por mi familia, y por mí primero.

Se comienza a producir una recuperación de la autoestima en situaciones sociales, sintiéndose más valorados en esos contextos:

- En la medida en que yo me fui sintiendo mejor, me fue dando más confianza como para hablar con más gente, porque cuando yo estaba consumiendo no podía hablar con más gente, porque yo sentía que todo el mundo me observaba. Pensaba que todo el mundo se iba a dar cuenta, "este tipo tomó o anda con olor a trago", y a lo mejor no lo tenía, pero yo sentía que sí... pero como yo ya no estaba consumiendo, me daba más confianza como para interactuar con los demás.

- Entonces yo ya notaba de que como que ya podía opinar, porque cuando yo consumía, yo te digo, no sé si es que yo me tiraba demasiado pa' abajo, porque yo no me atrevía ni a opinar, nada... a veces habían reuniones de trabajo y yo ni siquiera opinaba nada, y si alguien me preguntaba algo, trataba de dar cualquier respuesta para que no me preguntaran más... pero empecé como a opinar más, a tener más contacto con la gente.

- Yo a las tres semanas sin consumir, yo en mi casa me sentía como que me querían más, como yo no me sentía, o no me hacía la víctima.

A lo largo de las seis semanas comienzan a aparecer nuevas fuentes de gratificación, alternativas al consumo o a las actividades asociadas al consumo, que van enriqueciendo la vida de los sujetos:

- No estaba la de pisco o la de ron o la de vodka o lo que fuera, cachai, entonces empecé a pasarlo bien, por primera vez, cachai, después de un rato, y esta gente que en el fondo era súper sana... entonces empecé a pasarlo bien y eso me ayudó harto, ayuda harto.

- En las seis semanas me fui sintiendo cada día mejor, en las relaciones, yo como persona, cada día que pasaba me levantaba con más ganas. Cuando tomé el tema del gimnasio me empecé a hacer una especie de rutina, con lo poco y nada que tenía que hacer, y empecé a eliminar el tema del playstation.

- En algún minuto empecé a sentirme mejor, empecé a ver que me quedaba más tiempo, que tenía más plata, me empecé a entusiasmar con otras cosas, empecé a salir un poco de la rutina del carrete y ocupar bien mis sábados en la noche o las noches en otra cosa.

- Ocupar el tiempo que yo ocupaba consumiendo, en qué lo ocupo, eso también es re difícil cuando uno deja de consumir, porque el tiempo que tú ocupas consumiendo, después lo tienes libre, y tú dices "qué hago, qué hago en este rato"...Tenía como una hora y media, dos horas que eran de consumir, y después esas dos horas... ¿qué hago?". Entonces fue meterme más en el trabajo, trabajar más, quedarme hasta más tarde, o llegar a la casa más temprano.

Los distintos cambios que se van produciendo llevan a la emergencia de una nueva perspectiva con respecto al consumo de la sustancia y a un nuevo ángulo en relación a una identidad marcada por este consumo y sus consecuencias:

- Pensaba... imagínate que pensaba que mi abuelo, que falleció porque era alcohólico, y yo le echaba la culpa a eso, o sea, no la culpa pero yo decía bueno, si mi abuelo se murió así, quizás esta cuestión es así no más... Pero era una excusa, era algo que yo me estaba inventando pa' poder seguir consumiendo, o sea, hasta eso pensaba, esta cuestión es hereditaria, a mi abuelo le pasó, a mí también me va a pasar... Y no lo encontraba malo, mira la ridiculez, no lo encontraba malo... es como mi destino... y no poh. Después cuando me fui dando cuenta que podía... perfectamente vivir sin consumir, y después de todo ese tiempo como que volví a ser yo, en todo sentido.

- No ser capaz de lograr algo, eso es lo otro, cuando decían que no eres capaz, que nunca los proyectos los terminaste, y no terminar este otro proyecto es como incómodo... Yo creo que fue el tiempo de las seis semanas, de lograrlo, te proponen algo y tratar de lograrlo, de terminar un proyecto, fue que me dijeran "tú te propones un proyecto y no lo terminas nunca" y esto fue como empezar a hacer un proyecto que eran seis semanas... son proyectos que hay que cumplirlos... cuando dicen "ya, pasemos a la otra parte", yo ya estaba entregado, o sea, iba a hacer lo que me pidieran.

La nueva perspectiva que aparece lleva a preguntarse por el lugar en que se está en la vida en este momento:

- Entonces...fue... fue... igual fue doloroso, fue como si me sacaran la anestesia y empezar a ver qué onda... qué onda con todo, conmigo... empezar a mirar pa’ atrás, a ver dónde estaba.

- En ese sentido pa mí ese período de abstinencia fue fundamental... eh... hubo un cambio de... de posición, lógicamente, pero de un pasito, era como de dónde venía y de dónde estaba y de todo lo que había que hacer... Puta, era como... yo diría que siquiera de aquí a la puerta, o como que se me hiciera la imagen de que había una puerta, y de que se podía caminar por esa puerta y de que pasando esa huevá había otra huevá... pero como te digo, surge la idea de la puerta, del camino.

- Me empecé a deshacer de los libros, a hacer orden y recuento de todo lo que tenía... Tratar de decir fuera, 
empezar a deshacerme, empezar a ver con qué me quedo, con qué no me quedo, qué quiero llevarme de aquí pa allá, llevar fotos, o pasarlas.

- Yo creo que simplemente la esperanza de que las cosas podían ser diferentes.

- Igual, puta que me dolieron esas seis semanas, fueron tan... dolorosas, o sea, el darme cuenta de dónde estaba... cachai. Fue $\tan \tan \tan$ doloroso que no te dan ganas de volver atrás... No quería volver a pasar por ese dolor de nuevo... no iba a volver a pasar de nuevo... o sea, si ya había empezado no podía.

Las recaídas que se pueden producir durante el período de las seis semanas, también pueden tener un efecto positivo en términos de la decisión de tratarse:

- Porque al principio yo estaba súper seguro cuando empecé. “¿Cómo estai?” y yo no hablaba nada, decía "bien, súper bien, me siento la raja”... Y pum caí, y delante de todos, una vergüenza de decir que estaba súper bien y recaer fue como que uno no lo asumía que estaba mal cuando entras. Y a la semana, cuando me preguntan cómo estai digo "súper bien", a la segunda "súper bien 2, a la tercera "súper bien", a la cuarta "súper bien". Y, bueno, como me estoy engañando yo mismo, me estaba engañando yo mismo. Por eso, cuando recaí, me hizo el clic de, bueno, estoy súper mal. Y tenís que asumir lo que te están diciendo ellos, porque yo me sentaba, pero no escuchaba lo que hablaban, como que me daba lo mismo.

- Cuando recaí es como perder lo que ya había logrado... recaí y dije "estoy cagado, no he logrado nada, no he logrado nada nada nada, he sido mentiroso con todos los demás". Y no fui sincero, porque me preguntaban y yo decía "sí estoy bien" y ahí me quedaba, no tenía ganas de conversar, no era capaz de dialogar o de contar las cosas.

- Sabís lo que me dio más lata fue la vergüenza de haber recaído siendo que yo me había metido en la etapa de decir voy a salir de esto, eso fue lo que me dio, me dio vergüenza de no lograr algo, no sé poh, yo me propongo algo y trato de lograrlo y eso fue lo que me dio vergüenza, no poder salir...que me estaban apoyando todos.

- No pude estar tres semanas sin fumarme un pito, pero por lo menos hice un switch, por lo menos atiné, me metí y después igual paré y no recaí más, cachai.

- Pero realmente cuando yo me di cuenta que sí necesitaba el tratamiento fue cuando recaí... cuando recaí y me di cuenta que estaba mal, ahí yo dije ya necesito salir adelante... O sea implica que no era capaz de dominar mi propio cuerpo, eso fue lo que más me complicó, entonces eso fue lo más difícil pa mí...decir "ya, estoy mal" y eso fue realmente el principio de todo esto, me costó asumirlo, pero eso punto fue el que marcó ya la diferencia de decir "ya, tengo que seguir con esto".
Creo que de alguna manera me empecé a entusiasmar, y sentí que lo que estaba haciendo no estaba bien, sentía que tenía que parar, sentí que, me dije bueno tengo que hacerlo, o lo hago bien o no lo hago, cachai.

Las distintas vivencias relatadas por los sujetos referidas al período de intervención motivacional, facilitan la alternativa de que al final del proceso de seis semanas se tome una decisión de tratarse a más largo plazo:

- Que había mejorado la relación familiar, había mejorado el aspecto laboral, había mejorado mi estado físico... eso yo creo que... el haber vivido los cambios que te da el hecho de no estar consumiendo, que de todas maneras eran positivos, de todas maneras, o sea el no sentirse mal, no sentirse enfermo, no tener malestares estomacales, no tener dolores de cabeza, poder conversar con una persona sin tener miedo de que te sienta olor a trago, todas esas cosas en esas seis semanas... Entonces yo creo que esa fue la decisión que me... sentir que habían cosas que cambiaban, que yo era capaz.

- Con esa claridad tomar la decisión luego de seguir el tratamiento los dos años, o lo que fuera... hasta que me curara...O sea, hacer el tratamiento, meterme, no irme pa’ ningún lado.

- Yo creo que es fundamental esas seis semanas, lo poco que yo pude recopilar de mí o vivir en esas seis semanas fueron fundamentales pa poder tomar la decisión de tratarse, en el sentido de que veís lo mal que estai, lo mal que has estado toda tu vida, lo necesario y la urgencia huevón de arreglar esta huevá, la importancia que tiene a nivel vital el arreglar ese problema poh.

- Esas seis semanas me sirvieron para darme cuenta de que podía seguir el tratamiento, cuando llegué yo no sabía que podía seguir el tratamiento, y como te digo, pensando en engañarlos y todo... que son cambios súper positivos... y eso me dio valor para seguir después el tratamiento.

- Esas seis semanas son fundamentales yo diría, como para reafirmar la decisión de seguir el tratamiento, reafirmar la vida y yo creo de ahí seguir.

- Entonces, en el fondo era como alargar las seis semanas, era como alargarlas no más, si había que seguir avanzando, no había que hacer nada extra, [...] seguir sin consumo no más.

\section{Discusión}

En los resultados obtenidos se puede apreciar que el proceso de la IM, incluyendo la muestra de abstinencia, tiene un efecto positivo sobre la decisión de entrar a tratamiento de adicción, y sobre la generación de condiciones subjetivas 
para la entrada a un proceso psicoterapéutico. En el relato de los pacientes aparecen varios efectos producidos durante el período de seis semanas de la IM, mostrándose, a su vez, la concatenación de estos efectos. En primer lugar se puede destacar un bienestar directamente físico, relacionado con la desintoxicación. En estos relatos se releva el bienestar producido con la detención del consumo y la importancia de esto para los sujetos, más que el malestar por un síndrome de abstinencia. Parte de este bienestar se relaciona con la sensación de que los deseos de consumo comienzan a disminuir ya en estas primeras semanas. También es fuente de bienestar la impresión de recuperación de las facultades cognitivas que permite comenzar a tener mayor claridad mental para analizar la situación en que se está.

En segundo lugar, los sujetos le dan gran importancia al hecho de lograr detener el consumo de forma ambulatoria, es decir, de una forma que esta detención depende de su propia capacidad de mantener una decisión,y no de la voluntad de otros. El logro de esta abstinencia, que muchas veces ha sido intentada a lo largo de la historia de consumo en variadas ocasiones por los sujetos, comienza a producir un cambio en su autopercepción, en el sentido de sentirse capaces de relacionarse de un modo distinto con las sustancias adictivas. Esto les permite empezar a tener una visión más positiva de la posibilidad de enfrentar los problemas propios de la vida. Durante los años de consumo se va instalando la sensación de que no es posible hacerle frente a los deseos de consumo, lo que queda cuestionado en el período de la IM, abriéndose ahora un horizonte de posibilidad distinto.

En tercer lugar, los participantes relatan cambios significativos en la forma de relacionarse con los terceros significativos, en el caso de este estudio, sus familiares. Los sujetos, en la medida que se sienten mejor y tienen más claridad de la situación en que han vivido, comienzan a valorar de otro modo la preocupación mostrada por sus familiares. Esta preocupación comienza a ser vivida como un apoyo en lugar de una presión o una intrusión en sus vidas. A su vez, los familiares, al ver que el sujeto realiza un esfuerzo por mantener el acuerdo de abstinencia, comienzan a creer que esta vez sí podría estar comprometido con hacer un cambio en el ámbito de su consumo de sustancias. Además, también ven los cambios a nivel del adquirir una mayor claridad en el análisis de su situación, y a nivel de las relaciones afectivas. De esta manera, se empieza a recuperar una incipiente confianza en la disposición del miembro de la familia con el problema. Este cambio por parte de los familiares, a su vez, es percibido por los sujetos, lo que se convierte en un importante incentivo para seguir adelante con el proceso. Parte de esta recuperación de las confianzas mutuas también tiene que ver con una apertura de parte del potencial paciente, que para él adquiere la forma de un alivio por salir de una doble vida que le implicaba una cantidad enorme de esfuerzo mantener. Este cambio en el tipo de relación con los familiares también se extiende a otros lazos sociales, lo que les permite volver a sentirse dignos de estar en contextos sociales.

Todos los cambios nombrados funcionan retroalimentándose mutuamente, desarrollándose una verdadera cadena de cambios en distintos niveles, a partir del acuerdo de muestra de abstinencia. Esto lleva a que hagan su aparición, en forma creciente, fuentes alternativas de gratificación al consumo de sustancias que poco a poco permiten al sujeto ir ampliando su horizonte de vida. Dentro de estas nuevas fuentes de gratificación está la relación con las personas cercanas, lo que, nuevamente, retroalimenta los cambios positivos en ese ámbito. Las alternativas vitales vuelven a surgir de un modo más enriquecido, ayudando a ir saliendo de la monotonía propia del consumo adictivo. En directa relación con esto, el sujeto comienza a preguntarse por el lugar que ocupa en el mundo, el momento de vida en el que se encuentra, lo que abre la oportunidad a la realización de un trabajo psicoterapéutico que lo ayude a elaborar su historia y proyectarse hacia un futuro relacionándose de un modo más libre con sus deseos. En este sentido, la IM puede considerarse una intervención que intenta generar las condiciones para una actividad psicoterapéutica, además de fomentar la decisión de realizar un cambio significativo en el consumo de sustancias.

La consecuencia de los cambios descritos es que los entrevistados perciben la decisión de entrar a un tratamiento de adicción como una consecuencia natural de lo vivido durante esas primeras semanas. Lo viven simplemente como un seguir adelante con el proceso que ya se echó a andar en sus distintos niveles. La situación cambió, ellos quedaron ubicados en otro lugar real y simbólico, y esta es una situación que quisieran mantener para el futuro. La decisión de tratarse no parece vivirse como una gran decisión luego de este período, sino, más bien, como un continuar avanzando en la nueva dirección establecida. Así, según los relatos revisados, la decisión de entrar a tratamiento queda fuertemente facilitada por la IM.

Es llamativo lo que los participantes que recayeron durante estas seis semanas relatan. Las recaídas, que de todos modos involucran una baja muy sustantiva del consumo de sustancias comparado con el nivel de consumo antes de entrar a la IM, son vividas como algo que los obliga a tener que pronunciarse de manera más clara con respecto a la decisión involucrada en el acuerdo. Es decir, las recaídas, dado el contexto de acuerdo, de mayor desintoxicación, de mayor claridad, de mejoría en las relaciones con los familiares, etc., los interpelan como sujetos libres y capaces de tomar decisiones. Las características de la IM llevan a que el sujeto no se con- 
sidere un ente pasivo sobre el cual se interviene, sino que se vea como un sujeto activo y con derecho a decidir. Esto sería justamente lo inverso de lo que el sujeto ha sentido largamente en su relación con las sustancias adictivas.

Se podría plantear que la IM es un recurso que pretende encontrar un camino alejado tanto de un modelo médico (Foucault, 1963/1966), como de un psicologismo que ha tendido a teñir las aproximaciones psicoanalíticas sobre el tema durante más de un siglo (López, 2011b). Desde el modelo médico, el problema de la motivación a tratamiento siempre se contrapone con una necesidad de poder intervenir sobre un sujeto que es capaz, y además tiene el derecho, de decidir qué cambios realizar y qué tratamientos llevar a cabo. Esta forma de intervención requerida parece quedar fuera de los límites de ese modelo, el cual apela finalmente a la alternativa del simple, aunque muchas veces sutil, ejercicio de un poder (Foucault, 2003/2005). Por otra parte, desde el psicoanálisis, pero también desde muchas otras escuelas psicoterapéuticas, se ha tendido a entender el problema de la adicción a sustancias como el síntoma de alguna condición psicológica que debiera ser tratada para que entonces su síntoma amainara o desapareciera. El problema de estas aproximaciones es que no parecen tomar en cuenta la particularidad de una conducta que hace ingresar sustancias químicas que alteran de un modo muy real el funcionamiento del sistema nervioso y sus funciones. Esto lleva a que no se pueda considerar la adicción como un síntoma en el sentido psicológico del término, y que se haga necesaria una forma de intervenir que logre cortar una serie de retroalimentaciones involucradas en el proceso adictivo. Aquí es donde la aparente paradoja de la propuesta de detener el consumo como primer paso, para que el sujeto tenga la oportunidad de plantearse la posibilidad de detenerlo en el mediano y largo plazo, puede tener un lugar relevante en los abordajes motivacionales.

Finalmente, es necesario recalcar que este es un estudio que utiliza una metodología cualitativa que permite profundizar en el relato de las vivencias de los sujetos y que, por lo mismo, es limitado en sus posibilidades de generalización
(Stake, 2010; Yin, 2012). En este sentido habría que considerarlo un estudio preliminar.

\section{Conclusión}

A partir de este estudio se identifican varios efectos de cambio producidos en el período de seis semanas de duración de la intervención motivacional, intervención que tiene como elementos centrales la incorporación de terceros significativos y el acuerdo de participar en una serie de instancias terapéuticas, incluyendo el intento de detener totalmente el consumo de sustancias químicas en el contexto de una muestra de abstinencia. Entre estos efectos se puede destacar el logro de un bienestar físico y emocional, la mejoría de las capacidades cognitivas y claridad mental, la disminución de los deseos por consumir la sustancia, el aumento en la percepción de autoeficacia, la recuperación de la autoestima social, una mejoría importante en las relaciones familiares y las confianzas involucradas en ellas, y la aparición de fuentes de gratificación alternativas al consumo de sustancias. Estos cambios que se producen tienen una mutua influencia, potenciándose, y desencadenando cada vez cambios mayores. Al cabo de las seis semanas, la situación del sujeto y su entorno ha cambiado lo suficiente como para que la decisión de entrar a un tratamiento de adicción esté facilitada y sea vivida como algo natural. También se ha producido una apertura en el sujeto que promueve la posibilidad, en una segunda etapa, de un trabajo psicoterapéuticoanivel individual y/o familiar.

Aparece como especialmente relevante el que la IM se produzca en el contexto de un acuerdo entre el equipo terapéutico, el sujeto y sus terceros significativos. Esto lleva a que el sujeto se sienta como un participante activo del proceso, quedando interpelado en su posibilidad de decidir en relación a su consumo de sustancias. El contexto de haber realizado un acuerdo es también lo que facilita y promueve un cambio, inicial pero decisivo, en el tipo de relación que se entabla entre el sujeto y sus terceros significativos. El comienzo de un restablecimiento de las confianzas mutuas es destacable. 


\section{Referencias}

Beck, A., Wright, F., Newman, C. y Liese, B. (1999). Terapia cognitiva de las drogodependencias (A. Rodríguez, Trad.). España: Paidós. (Trabajo original publicado en 1993).

Cade, B. y O’Hanlon, W. H. (1995).Guía breve de terapia breve. Barcelona: Paidós.

Carpenter, K., Miele, G. y Hasin, D. (2002). Does motivation to change mediate the effect of DSM-IV substance use disorders on treatment utilization and substance use? Addictive Behaviors, 27, 207-225. doi: 10.1016/S0306-4603(00)00178-7

Cunningham, J. y Breslin, F. (2004). Only one in three people with alcohol abuse or dependence ever seek treatment. Addictive Behaviors, 29, 221-223.doi: 10.1016/S0306-4603(03)00077-7

DiClemente, Ph., Bellino, L. y Neavins, T. (1999). Motivation for change and alcoholism treatment.Alcohol Research \& Health, 23, 86-92.

Downey, L., Rosengren, D. y Donovan, M. (2001). Sources of motivation for abstinence: A replication analysis of the Reasons for Quitting Questionnaire. Addictive Behaviors, 26, 79-89.

Flick, U. (2000). Episodic interviewing. En M. Bauer y G. Gaskell (Comps.), Qualitative Researching with text, image and sound: A practical handbook (pp. 75-92). Londres: Sage.

Flick, U. (2004). Introducción a la investigación cualitativa (P. Manzano, Trad.). Madrid: Editorial Morata. (Trabajo original publicado en 2002).

Foucault, M. (1966). El nacimiento de la clínica (F. Perujo, Trad.). México: Siglo Veintiuno Editores. (Trabajo original publicado en 1963).

Foucault, M. (2005). El poder psiquiátrico (H. Pons, Trad.). Buenos Aires: Fondo de Cultura Económica. (Trabajo original publicado en 2003).

Heyman, G. M. (2009). Addiction: A disorder of choice. Cambridge, MA: Harvard University Press.

Johnson, V. (1973). I'll quit tomorrow. New York: Harper \& Rowe.

Kaskutas, L. A., Subbaraman, M. S., Witbrodt, J. y Zemore, S. E. (2009). Effectiveness of making Alcoholics Anonymous easier: A group format 12-step facilitation approach. Journal of Substance Abuse Treatment, 37, 228-239. doi: 10.1016/j. jsat.2009.01.004

Kreither, J. y López, C. (2001).Tratamiento de adicciones: una forma de comenzar. Psykhe, 10(2), 11-22.

Landau, J., Stanton, D., Brinkman-Sull, D., Ikle, D., McCormick, D., Garrett, J., ...Wamboldt, F. (2004). Outcomes with the ARISE approach to engaging reluctant drug- and alcohol-dependent individuals in treatment. American Journal of Drug and Alcohol Abuse, 30, 711-748. doi: 10.1081/ADA-200037533

Lee, M. T., Garnick, D. W., O’Brien, P. L., Lee, P., Ritter, G. A., Acevedo, A.,...Godley, M. D. (2012). Adolescent treatment initiation and engagement in anevidence-based practice initiative. Journal of Substance Abuse Treatment, 42, 346-355. doi: $10.1016 /$ j.jsat.2011.09.005
Lincourt, P., Kuettel, T. y Bombardier, C.H. (2002). Motivational interviewing in a group setting with mandate clients: A pilot study. Addictive Behaviors, 27, 381-391.

López, C. (2006). La adicción a sustancias químicas: ¿Puede ser efectivo un abordaje psicoanalítico? Psykhe, 15(1),67-77.

López, C. (2009). La decisión de entrar a un tratamiento de adicciones: Motivación propia e influencia de terceros. Terapia Psicológica, 27(1), 119-127. doi: 10.4067/S071848082009000100012

López, C. (2011a). Las características de la situación mental en el consumo adictivo de sustancias y la motivación para entrar a un tratamiento de adicciones. Revista de Psicología, 20(2), 53-76.

López, C. (2011b). Adicción a sustancias químicas: ¿Enfermedad primaria o síntoma psicoanalítico? Praxis, 20, 41-60.

Meyers, R. J., Roozen, H. G. y Smith, J. (2011). The community reinforcement approach: An update of the evidence. Alcohol Research \& Health, 33(4), 380-388.

Meyers, R. J. y Smith, J. (1995). Clinical guide to alcohol treatment. New York: Guilford Press.

Miller, W., Forcehimes, A. y Zweben, A. (2011). Treating addiction: A guide for professionals. New York: Guilford Press.

Miller, W. y Meyers, R. (1999). The community-reinforcement approach. Alcohol, Research and Health, 23(2), 116-122. doi: 10.1017/CBO9780511570117.006

Miller, R. y Page, A. (1991). Warm turkey: Other routes to abstinence. Journal of Substance Abuse Treatment, 8, 227-232. doi: 10.1016/0740-5472(91)90043-A

Miller, W. y Rollnick, S. (1999). La entrevista motivacional (A. Tejero, Trad.). España: Paidós. (Trabajo original publicado en 1991).

Nardone, G. y Watzlawick, P. (1992). El arte del cambio (A. Martínez-Riu, Trad.). Barcelona: Herder. (Trabajo original publicado en 1990).

Santisteban, D., Suárez-Morales, L., Robbins, M. y Szapocznik, J. (2006). Brief strategic family therapy: Lessons learned in efficacy research and challenges to blending research and practice. Family Process, 45, 259-271. doi: 10.1111/j.15455300.2006.00094.x

Stake, R. (2010). Qualitative research. New York: Guilford Press.

Stinson, F. S., Grant, B. F., Dawson, D. A., Ruan, W. J., Huang, B. y Saha, T. (2005). Comorbidity between DSM-IV alcohol and specific drug use disorders in the United States: Results from the National Epidemiologic Survey on Alcohol and Related Conditions. Drug and Alcohol Dependence, 80(1), 105-16. doi: 10.1016/j.drugalcdep.2005.03.009

Substance Abuse and Mental Health Services Administration, Office of Applied Studies (SAMHSA). (2009). The NSDUH report-alcohol treatment: Need, utilization, andbarriers. Rockville, MD.

Tatarsky, A. (2003). Harm reduction psychotherapy: Extending the reach of traditional substance use treatment. Journal of Substance Abuse Treatment, 25(4), 249-256. doi: 10.1016/ S0740-5472(03)00085-0 
Timko, C. y DeBenedetti, A. (2007). A randomized controlled trial of intensive referral to 12-step self-help groups: One-year outcomes. Drug and Alcohol Dependence, 90(2-3), 270-279. doi: 10.1016/j.drugalcdep.2007.04.007

Ulivi, G. (2000). Terapia breve centrada en la solución como modelo de terapia familiar de toxicómanos. Adicciones, 12(3), 425-430.
Watzlawick, P. y Nardone, G. (2000). Terapia breve estratégica. Barcelona: Paidós.

Wild, C., Cunningham, J. y Ryan, R. (2006). Social pressure, coercion, and client engagement at treatment entry: A self-determination theory perspective. Addictive Behaviors, 31(10), 1858-1872.

Yin, R. (2012). Case study research. USA: SAGE Publications.

Fecha de recepción: 30 de abril de 2013 Fecha de aceptación: 17 de julio de 2013 